\title{
Beyond the paper
}

We invite researchers to make use of our community site to disseminate their research and foster discussion.

$\mathrm{L}$ aunched at the same time as Nature Ecology \& Evolution, the Nature Portfolio Ecology \& Evolution Community is a blog site for informal discussion of research and other scientific community news. There is a range of channels in which members can post, with the Behind the Paper channel being the overwhelming favourite. Usually posted at the same time as publication of the paper, these posts allow authors to tell their research story, including the genesis of their ideas, interactions with their collaborators, and what went right or wrong in the lab or field. It is also a great place to showcase images of the organisms studied or the researchers in action. So far, over 1,000 authors have taken this opportunity to share such experiences - the channel therefore represents a wealth of fascinating stories. The posts allow colleagues and the wider public to understand a bit more of the human side of doing research. In the coming months, for example, we might expect to see authors describing how COVID-19 lockdowns have affected their projects and what they have had to do to adapt.

But the story of a paper does not end with publication. The main purpose of publishing a peer-reviewed version of record is to share the results broadly, with the aim of influencing future research and policy, as well as the authors' own careers. We've therefore recently launched an After the Paper channel, in which authors can return to their study a year or so on and tell readers of its consequences. These might include engagement with a wider range of stakeholders than the usual academic and policy circles, such as local communities. The few examples posted to date include diversity, equity and inclusion initiatives arising from a study of the disproportionate contributions to such initiatives by underrepresented faculty, engaging with policymakers in Gabon on the basis of a study of carbon storage caused by elephants, and making heard the voices of researchers from the Global South. We really value these thoughtful follow-ups to research studies, and encourage all authors to consider posting one.

While the majority of Behind and After the Paper posts come from authors of studies published in Springer Nature journals, some have not and it is definitely not a requirement. We encourage any authors of ecology or evolution manuscripts to make use of the site. There is also a range of other channels. The Journal Club section allows researchers and editors to discuss recent or classic papers from others. One possibility is to make use of this to share the outcomes of group or institutional journal club discussion meetings with a wider audience who might benefit. The On the Road channel includes both our annual list of ecology and evolution conferences and meeting reports from the editors. We also encourage meeting reports from researchers, from both virtual and in-person meetings. Finally, there are channels devoted to specific topics, such as COVID-19 or anti-racism, and specific events and anniversaries, such as World Oceans Day. If you would like to suggest such a channel, please do get in touch (ecoevo@nature.com).

It's important to note that there is no expectation on authors to contribute to the community site. We appreciate how many competing demands researchers have on their time, that different projects will be suited to different types of related content, and that there is a range of other platforms that can be used. We just encourage authors to use the community site where it is of benefit to them, and in conjunction with other ways of sharing their ideas and experiences.

Published online: 6 April 2021 https://doi.org/10.1038/s41559-021-01446-8 